\title{
Different Modes of Vancomycin and D-Alanyl-D-Alanine Peptidase Binding to Cell Wall Peptide and a Possible Role for the Vancomycin Resistance Protein
}

\author{
JAMES R. KNOX ${ }^{1 *}$ AND R. F. PRATT ${ }^{2}$ \\ Department of Molecular and Cell Biology, The University of Connecticut, Storrs, Connecticut 06269-3125, ${ }^{1}$ and \\ Department of Chemistry, Wesleyan University, Middletown, Connecticut $06457^{2}$
}

Received 26 February 1990/Accepted 11 April 1990

\begin{abstract}
A comparison was made of the binding modes of the bacterial cell wall precursor L-lysyl-D-alanyl-D-alanine to the glycopeptide antibiotic vancomycin and to the D-alanyl-D-alanine-cleaving peptidase of Streptomyces sp. strain R61, a model for cell wall-synthesizing enzymes whose X-ray three-dimensional structure is established. In each of the two pairings (vancomycin with peptide and DD-peptidase with peptide), polypeptide backbones were antiparallel, and the antibiotic or enzyme enveloped the peptide substrate from opposite sides. Hydrogenbonding groups on the substrate which are involved with the DD-peptidase were shown to be different from the ones reported from nuclear magnetic resonance studies to be involved with vancomycin. Because of steric hindrance, the binding of either molecule to the substrate prevents the binding of the other molecule. Binding to the substrate by a D-alanyl-D-alanine-recognizing protein in a manner similar to that used by the DD-peptidase could explain recent observations of vancomycin resistance, in which a new membrane-associated protein has been detected.
\end{abstract}

Glycopeptide antibiotics of the vancomycin family (including ristocetin, avoparcin, and teicoplanin) are increasingly used against gram-positive bacteria, especially those resistant to $\beta$-lactams. Like the $\beta$-lactams, the glycopeptides inhibit the synthesis of bacterial cell wall peptidoglycan. However, they inhibit synthesis not by inactivating the biosynthetic enzymes but, rather, by noncovalent binding to the D-alanyl-D-alanine (D-ala-D-ala) terminus of a pentapeptide cell wall precursor. It is unclear whether the antibiotics prevent transmission of the disaccharide pentapeptide monomer across the inner membrane to the region of the Dalanyl carboxypeptidases and transpeptidases (DD-peptidases) or whether the binding of antibiotic to the peptide occurs on the outer side of the membrane where direct competition with the D-ala-D-ala-recognizing enzymes is likely $(14,22)$.

Structural aspects of the complexation of vancomycin and ristocetin with the tripeptide terminus have been probed by nuclear magnetic resonance (NMR) spectroscopy $(2,19,20)$, and kinetic studies of the complexation have been reported $(16,19)$. With the recent determination of an X-ray crystal structure of a DD-peptidase and the modeling of tripeptide to the binding site of the enzyme (6), it is possible to ask whether a DD-peptidase and the much smaller vancomycintype antibiotics interact with a common cell wall substrate in a similar fashion. The DD-peptidase-substrate interaction could also serve as a model for vancomycin resistance $(9,11$, $18,21)$, in which a new $D$-ala-D-ala-recognizing protein may bind to the peptide terminus of the monomer at some point on its way to becoming a cross-linked peptidoglycan.

\section{MATERIALS AND METHODS}

Three-dimensional structures of vancomycin and DD-peptidase. In the absence of an X-ray crystallographic structure of

\footnotetext{
* Corresponding author.
}

vancomycin (Fig. 1), one must build vancomycin from the crystal structure of a degradation product, CDP-I, in which an asparagine at position 3 has been converted to isoaspartic acid. The CDP-I structure has been published previously (17), and its coordinates are available at the Brookhaven Protein Structure Data Bank (Upton, N.Y.). Initial coordinates of a vancomycin model were kindly provided by Robert Sheridan (Lederle Laboratories, Pearl River, N.Y.). They were derived from the X-ray structure of the CDP-I analog, and energy was minimized in the presence of $N$ acetyl-D-ala-D-ala. For the present study, this starting vancomycin model was altered at the $\mathrm{N}$ terminus and fitted to $\mathrm{L}$ lysyl-D-ala-D-ala to agree with NMR results $(2,19,20)$. The interactive fitting program FRODO (4) was used on a graphics system (PS330; Evans \& Sutherland). The X-ray crystallographic structures of the penicillin-sensitive, 38-kilodalton DD-peptidase of Streptomyces sp. strain R61 and five of its $\beta$-lactam complexes have been experimentally mapped at resolutions of $2.3 \AA(0.23 \mathrm{~nm})$ and $2.8 \AA(0.28 \mathrm{~nm})(6,7)$. Based on these maps, the binding of the L-lysyl-D-ala-D-ala substrate to the catalytic site of the enzyme has been modeled previously (6). Atomic coordinates of the complexes described below have been deposited at the Brookhaven Data Bank.

\section{RESULTS}

Interaction of the DD-peptidase with the cell wall peptide. Analysis of the atomic-level interactions between the DDpeptidase and the tripeptide substrate (6) shows that important features are, first, an antiparallel hydrogen bonding with $\beta$-strand b3 (residues 298 to 303) of the peptidase (Fig. 2A). In particular, the backbone amide $\mathrm{NH}$ and carbonyl groups of Thr-301 bind to the amide carbonyl and NH of the penultimate $\mathrm{D}$-alanine. The carbonyl of this $\mathrm{D}$-alanine forms a second hydrogen bond with the amide NH of Ser-62 on the adjacent a2 helix (Fig. 2B). The resulting polarization of the 


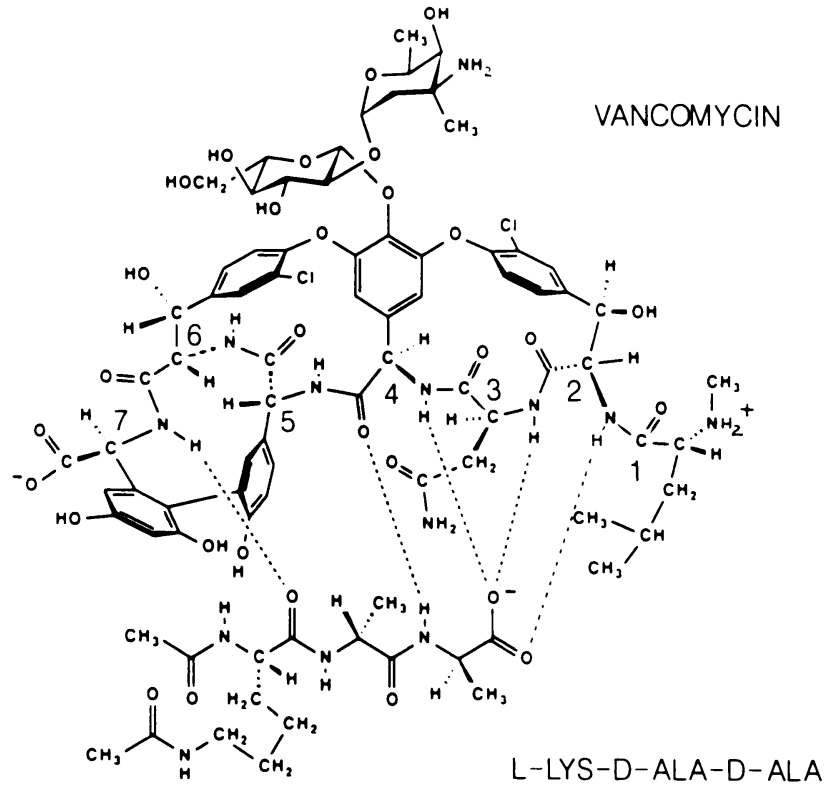

FIG. 1. Chemical structure of vancomycin, showing NMR-assigned interactions with diacetyl-L-lysyl-D-alanyl-D-alanine substrate (adapted from Kannan et al. [5]).

carbonyl bond of the substrate facilitates nucleophilic attack by the Ser-62 hydroxyl group. A second feature of the binding is the immobilization of the C-terminal carboxylate group of the peptide via interactions with the imidazole group of His-298, the hydroxyl group of Thr-299, and, possibly, the positively charged side chain of Lys-65 on the a2 helix. The enzymatic environment of the two D-methyl substituents is generally hydrophobic, with the penultimate methyl group being somewhat more buried. The L-lysyl side chain lies in a hydrogen-bonding and negatively charged region containing Thr-227, Glu-228, and Gln-303. The remainder of the peptidoglycan is expected to extend outward from Fig. 2, with the oligosaccharide running horizontally along the bottom of the enzyme (see also Fig. 3C).

Interaction of vancomycin with the cell wall peptide. Two schemes are considered for the interaction between the two molecules. One model (Fig. 3A) is derived from the fact that vancomycin and DD-peptidase have two structural features in common. (i) Both molecules contain an extended peptide chain which is aligned with substrate in a hydrogen-bonding array, and (ii) both molecules contain a protonated amine function which, in the antiparallel alignment with substrate, falls near the carboxylate group of the substrate (compare Fig. 2A and 3A). The model shown in Fig. 3A does not agree, however, with published NMR assignments for the vancomycin-substrate complex $(2,19,20)$, especially with regard to the hydrogen-bonded atoms indicated in Fig. 1. An alternative model for the interaction of the two molecules with the NMR-assigned hydrogen bonds found previously $(2,19,20)$ is shown in Fig. 3B. Specifically, the carbonyl of residue 4 of vancomycin and the amide $\mathrm{NH}$ of residue 7 bind to the C-terminal D-alanyl amide $\mathrm{NH}$ and the lysyl carbonyl of the substrate, respectively. Here, too, the two peptide strands align in an antiparallel fashion. Another feature in the binding is a shielding of the ionized carboxylate group of the substrate by the N-terminal D-leucyl portion of vancomycin, the result of which is optimization of the electrostatic

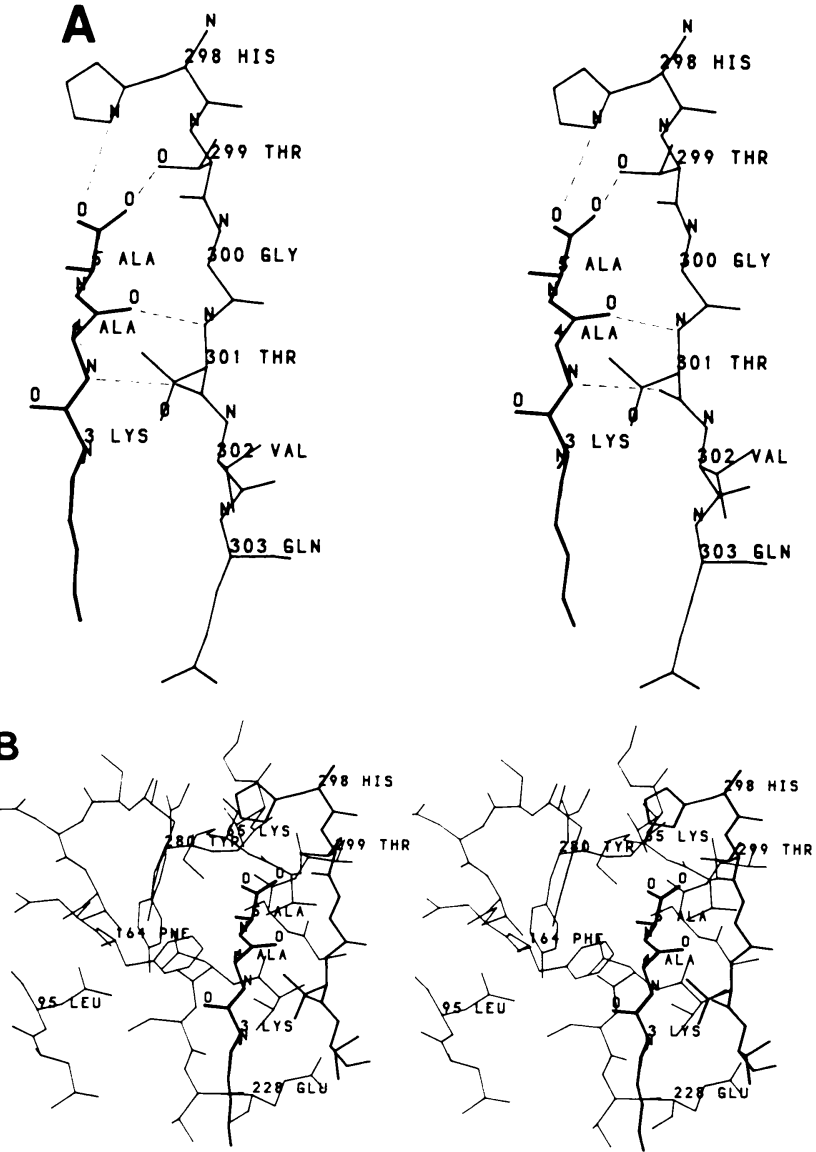

FIG. 2. (A) Stereoview of the $\beta$-strand b3 (residues 298 to 303 ) of the DD-peptidase of Streptomyces strain R61 aligned with L-lys-Dala-D-ala having the conformation taken from Kelly et al. (6), with $(\phi, \psi)$ equal $\left(-, 96^{\circ}\right),\left(145^{\circ},-120^{\circ}\right)$, and $\left(80^{\circ}, 30^{\circ}\right)$ from the $N$ to the $\mathrm{C}$ terminus. Hydrogen atoms are not shown. (B) Expanded stereoview of the binding site for the L-lysyl-D-ala-D-ala substrate (or $\beta$-lactam inhibitor) in the DD-peptidase of Streptomyces strain R61 (6).

attraction of the carboxylate to the three backbone amide $\mathrm{NH}$ groups of residues 2, 3, and 4. Recent data suggest that the methylated N-terminal amine becomes solvated by water as a hydrophobic cavity is formed around the substrate $(5$, 20). Thus, in the NMR-compatible model shown in Fig. 3B, the D-leucyl amine is directed away from the carboxylate group of the substrate.

The vancosamine sugar helps direct the specificity of the antibiotic for a D-configured methyl group on the C-terminal alanine (20). We propose that a role of the entire di- to hexasaccharide unit found in these glycopeptide antibiotics might be to help orient the peptide backbone of the antibiotic alongside the substrate backbone (Fig. 3C). Because the long axis of the oligosaccharide is generally perpendicular to the peptide backbone (as seen in the X-ray structure of CDP-I [17]), and because the $N$-acetylmuramic acid- $N$-acetylglucosamine oligosaccharide axis in peptidoglycan is likely to be perpendicular to the pentapeptide (as proposed from conformational modeling studies [12]), if the two glycopeptides were brought together, it would place hydroxyl groups of the saccharides within hydrogen-bonding distance. This mutual alignment of the two saccharide units and the two 

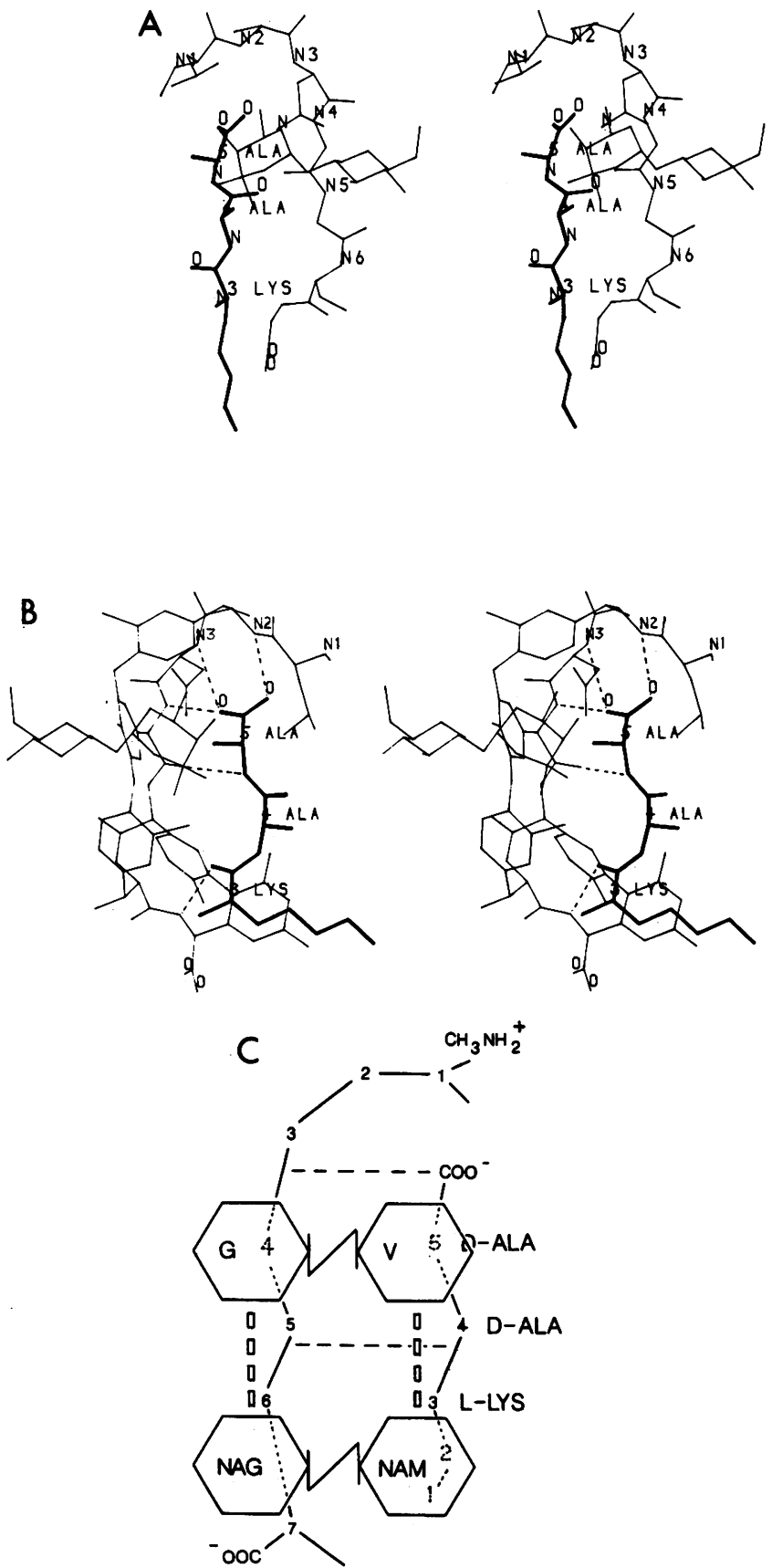

FIG. 3. (A) A model for the vancomycin-substrate interaction derived from the interaction of the substrate with the DD-peptidase (6), as diagrammed in Fig. 2A. For clarity, only the heptapeptide backbone and disaccharide of vancomycin are drawn. Hydrogen atoms are also omitted. (B) A model of the vancomycin-substrate complex based on previous NMR-derived proposals $(19,20)$, with solvation of the methylated N-terminal D-leucyl amine. (C) A possible mutual alignment of saccharide units and peptide units in vancomycin-peptidoglycan binding. Dashed lines and strips indicate directions of hydrogen binding. Glucosylvancosamine (G-V) is joined via the glucosyl (G) to the phenolic side chain of position 4 in vancomycin (left). $N$-Acetylglucosamine (NAG) and $N$-acetylmuramic acid (NAM) are joined via $N$-acetylmuramic acid to the pentapeptide substrate (right). peptide units could initiate complexation, stabilize complexation, or both. The attractiveness of such an alignment (Fig. 3C) further supports the peptide-to-peptide binding mode shown in Fig. 3B over that in Fig. 3A, in which the disaccharide of the antibiotic would be unable to hydrogen bond the $N$-acetylmuramic acid- $N$-acetylglucosamine oligosaccharide.

\section{DISCUSSION}

Comparison of the interactions. Figure $4 \mathrm{~A}$ shows that the antibiotic and enzyme each contain an oligopeptide of five to seven residues in length which aligns antiparallel to and along opposite sides of a common substrate. Each molecule uses at least two hydrogen bonds to bind to the backbone of the substrate, and the enzyme uses a third hydrogen bond from its a2 helix. Somewhat different modes are used for the interaction of each molecule with the C-terminal carboxylate group of the substrate. The enzyme surrounds the anion with three charged or hydrogen-bonding groups (Lys-65, His-298, Thr-299). In addition, a weak field of positive charge is generated in the carboxylate-binding area by the dipole moment of the long helix beginning at Ser-62. Between the $\mathbf{N}$ terminus of this helix and the $\beta$-strand b3 is an oxyanion hole which immobilizes the carbonyl group of the cleavable peptide bond of the D-ala-D-ala substrate.

The smaller vancomycin has fewer options available for interacting with the carboxylate of the substrate. The secondary amine of the $\mathrm{N}$-terminal D-leucine of vancomycin may be the counterpart of the conserved amine (usually a lysine or, in this case, His-298) of the cell wall-synthesizing enzymes (6), and one would expect an electrostatic interaction between the leucyl-ammonium ion and the carboxylate. In the NMR model, however, the ammonium ion is turned away from the carboxylate and is exposed to solvent in order to fold the $N$-methyl and leucyl groups over the carboxylate group and to involve the backbone amides of residues 2 and 3 in hydrogen bond interactions with the carboxylate $(5,19$, 20). In contrast, the DD-peptidase uses no backbone amide $\mathrm{NH}$ bonds for the carboxylate interaction. Because the binding site of the enzyme is not particularly deep or solvent inaccessible (Fig. 2B), His-298 may be solvated in the substrate-free enzyme, but any solvent is displaced by the carboxylate of the substrate. To maximize the binding energy with the substrate, the enzyme is presumably able to make use of numerous other interactions which are not available to the smaller vancomycin molecule. Nevertheless, the $K_{m}$ (and $K_{s}$ ) of the enzyme-substrate complex is several orders of magnitude larger than the dissociation constant of the vancomycin-substrate complex $(3,14)$. This difference presumably reflects the requirements for rapid catalysis and turnover in the former case versus the need for tight binding in the latter case.

The glycopeptide antibiotics, unlike DD-peptidases, do not strongly bind $D$-ala-D-ala analogs such as penicillins $(13,15)$, probably because of the inability of the $\beta$-lactam nitrogen atom to be a hydrogen bond donor and because of unfavorable steric interactions between the thiazolidine ring of a penicillin and the phenolic rings of glycopeptide at positions 2,4 , and 6 . It is interesting that the structural differences between peptide and penicillin, which in part preclude binding of the latter to the glycopeptide antibiotics, are not more effectively used by DD-peptidases to resist $\beta$-lactams. Presumably, the structural changes required by catalysis, viz., the change in geometry at the amide nitrogen on formation of the tetrahedral intermediate and the need to 
A
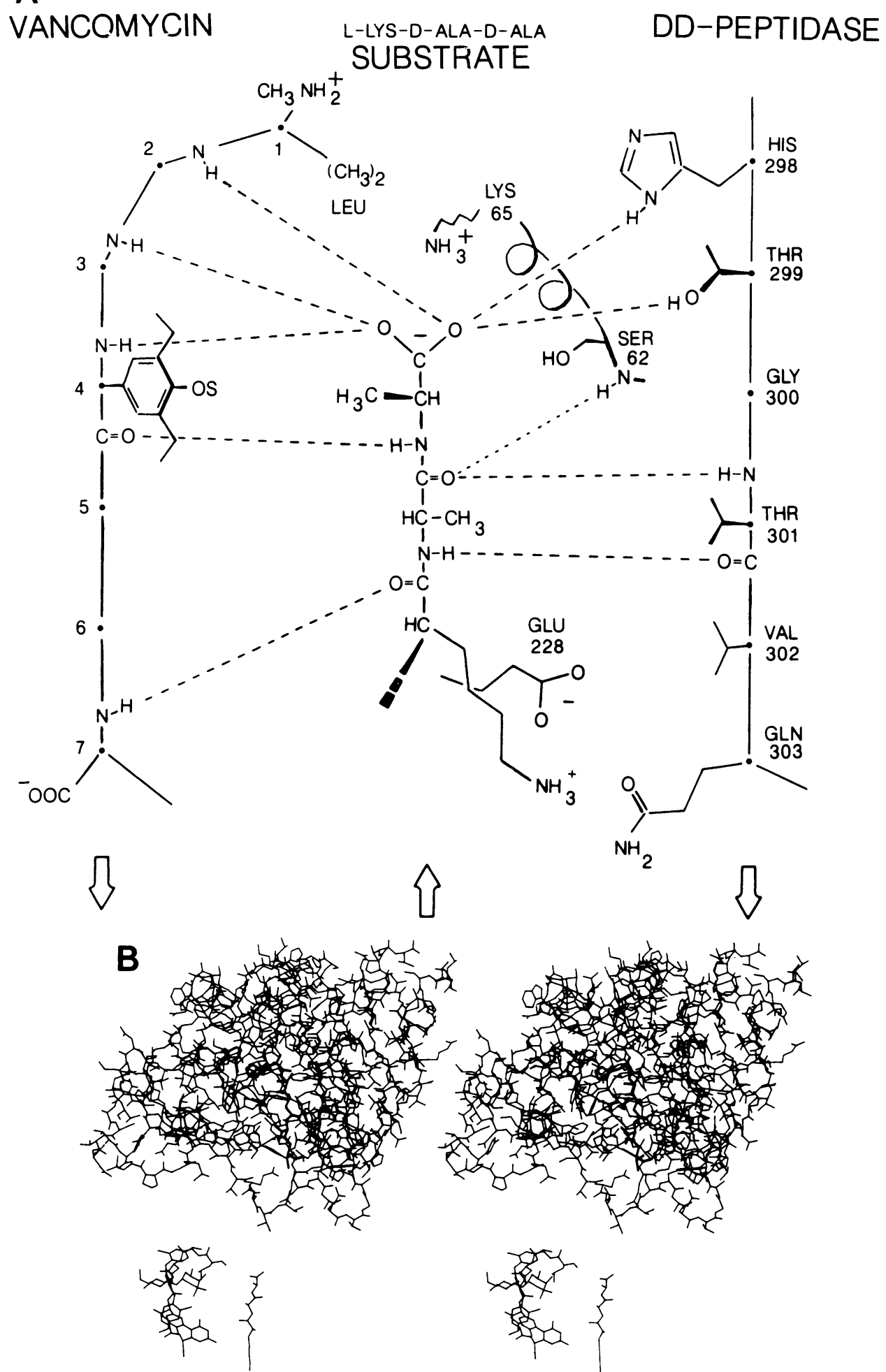

FIG. 4. (A) Juxtaposition of the mutually exclusive binding modes used by vancomycin (left) or DD-peptidase (right) for interaction with their common substrate. Hydrogen bonds are indicated by dashes; distances are not to scale. Arrows show direction of adjacent polypeptides. (B) Stereoview showing the relative sizes of vancomycin (1.5 kilodaltons), the DD-peptidase (38 kilodaltons) of Streptomyces strain R61, and their tripeptide substrate. Because of steric hindrance, the binding of one to the substrate would exclude the binding of the other. Molecules are oriented as shown in Fig. 2 and 3B. 
bind the acyl group acceptor, do not allow such discrimination.

DD-Peptidase and the vancomycin resistance protein. Inducible resistance to glycopeptide antibiotics has recently been discovered in various Enterococcus species $(9,11,18,21)$. The resistance appears to be associated with a transferable plasmid which carries the gene for a new 39- to 40-kilodalton membrane-associated protein. This protein does not appear to destroy the antibiotics (21). Rather, it is reported to be homologous (38\%) to a D-ala-D-ala ligase (S. Dutka-Malen, A. Brisson-Noel, C. Molinas, and P. Courvalin, Program Abstr. 29th Intersci. Conf. Antimicrob. Agents Chemother., abstr. no. 272, 1984), an observation which suggests several possibilities for the resistance mechanism.

First, the resistance protein may be another ligase. The resistance could then arise from an increased supply of $D$ ala-D-ala fragments. Since the binding of acyl-D-ala-D-ala species to glycopeptide antibiotics is not controlled by diffusion (16) and may be slower than binding to DDpeptidases, an increased rate of synthesis of these decoy fragments may allow their steady-state concentration to be sufficiently high (although low with respect to that under normal antibiotic-free conditions) to prevent cell lysis.

Shlaes and co-workers (18) have broadly theorized that the resistance protein prevents access of the antibiotics to their peptidoglycan targets. Based on the aforementioned NMR and crystallographic data, which indicate that vancomycin and DD-peptidase envelop the peptide from opposite sides, our analysis shows that steric factors would prevent mutual binding of the three molecules (Fig. 4B). Herein may lie an alternative molecular mechanism of resistance to vancomycin. The ligase-like resistance protein may noncovalently bind the D-ala-D-ala termini in a manner similar to that used by the DD-peptidase described here. The normal ligase does bind D-ala-D-ala, the product of its reaction, although not strongly $\left(K_{i}, \sim 1 \mathrm{mM}\right)(10)$. The ligase shows no obvious homology with the strain R61 DD-peptidase, but an $\mathrm{X}$-ray determination of the ligase tertiary structure, which is in progress (8), may reveal a common architecture at the substrate- or product-binding site. The resistance protein, unlike the normal ligase, is bound to the cytoplasmic membrane $(9,11,18,21)$. It may therefore serve to collect peptidoglycan monomers inside the cell and facilitate their transmission through the membrane. On the outer side of the membrane, the resistance protein might then release the monomer units (very tight binding would be counterproductive) close enough to the DD-peptidase to allow sufficient transglycosylase-transpeptidase activity to maintain the cell wall. It is possible that much of the undecaprenyl phosphate, the normal carrier, may be tied up in monomer-antibiotic complexes. Unlike the undecaprenyl phosphate-monomer complex, the resistance protein-monomer complex would not be sequestered by antibiotic because the resistance protein and the antibiotic, like the DD-peptidase and the antibiotic, may not be able to bind to a peptidoglycan monomer unit simultaneously.

That the resistance protein and the DD-peptidase may bind the cell wall peptide in similar fashion is supported by a recent report (1) that appeared after this article was submitted. From their data showing the time-dependent disappearance of glycopeptide binding to the pentapeptide after treatment with membranes from resistant cells, Al-Obeid et al. (1) have proposed that the resistance protein functions as a DDpeptidase.

\section{ACKNOWLEDGMENTS}

We are indebted to Robert Sheridan for generously providing coordinates of a vancomycin model; and we thank N. E. Allen, T. I. Nicas, D. J. Tipper, and R. Williamson for helpful discussions.

\section{LITERATURE CITED}

1. Al-Obeid, S., E. Collatz, and L. Gutmann. 1990. Mechanism of resistance of vancomycin in Enterococcus faecium D366 and Enterococcus faecalis A256. Antimicrob. Agents Chemother. 34:252-256.

2. Fesik, S. W., T. J. O'Donnell, R. T. Gampe, and E. T. Olejniczak. 1988. Determining the structure of a glycopeptide-ac ${ }_{2}$-LysD-ala-D-ala complex using NMR parameters and molecular modeling. J. Am. Chem. Soc. 108:3165-3170.

3. Frere, J. M., and B. Joris. 1985. Penicillin-sensitive enzymes in peptidoglycan biosynthesis. Crit. Rev. Microbiol. 11:299-396.

4. Jones, T. A. 1985. Interactive computer graphics: FRODO. Methods Enzymol. 115:157-171.

5. Kannan, R., C. M. Harris, T. M. Harris, J. P. Waltho, N. J. Skelton, and D. H. Williams. 1988. Function of the amino sugar and N-terminal amino acid of the antibiotic vancomycin in its complexation with cell wall peptides. J. Am. Chem. Soc. 110:2946-2953.

6. Kelly, J. A., J. R. Knox, H. Zhao, J. M. Frere, and J. M. Ghuysen. 1989. Crystallographic mapping of $\beta$-lactams bound to a DD-peptidase target enzyme. J. Mol. Biol. 209:281-295.

7. Knox, J. R., J. A. Kelly, P. C. Moews, H. Zhao, J. Moring, J. K. M. Rao, J. C. Boyington, O. Dideberg, P. C. Charlier, and M. Lipert. 1987. Crystallography of penicillin-binding enzymes, p. 64-82. In Y. Iitaka and A. Itai (ed.), Three dimensional structure and drug action. University of Tokyo Press, Tokyo.

8. Knox, J. R., H. Liu, C. T. Walsh, and L. E. Zawadzke. 1989. D-Alanine: D-alanine ligase (ADP) from Salmonella typhimurium. Over-production, purification, crystallization and preliminary $\mathrm{x}$-ray analysis. J. Mol. Biol. 205:461-463.

9. Leclercq, R., E. Derlot, J. Duval, and P. Courvalin. 1988. Plasmid-mediated resistance to vancomycin and teicoplanin in Enterococcus faecium. N. Engl. J. Med. 319:157-161.

10. Neuhaus, F. C., C. V. Carpenter, J. M. Miller, N. M. Lee, M. Gragg, and R. A. Stickgold. 1969. Enzymatic synthesis of Dalanyl-D-alanine. Control of D-alanine: D-alanine ligase (ADP). Biochemistry 8:5119-5123.

11. Nicas, T. I., C. Y. E. Wu, J. N. Hobbs, D. A. Preston, and N. E. Allen. 1989. Characterization of vancomycin resistance in Enterococcus faecium and Enterococcus faecalis. Antimicrob. Agents Chemother. 33:1121-1124.

12. Oldmixon, E. H., S. Glauser, and M. L. Higgins. 1974. Two proposed general configurations for bacterial cell wall peptidoglycans shown by space-filling molecular models. Biopolymers 13:2037-2060.

13. Perkins, H. R. 1969. Specificity of combination between mucopeptide precursors and vancomycin or ristocetin. Biochem. J. 111:195-205.

14. Perkins, H. R. 1985. Vancomycin and related antibiotics, p. 115-132. In D. J. Tipper (ed.), Antibiotic inhibitors of bacterial cell wall biosynthesis. International encyclopedia of pharmacology and therapeutics. Pergamon Press, Oxford.

15. Popieniek, P. H., and R. F. Pratt. 1987. A fluorescent ligand for binding studies with glycopeptide antibiotics of the vancomycin class. Anal. Biochem. 165:108-113.

16. Popieniek, P. H., and R. F. Pratt. 1988. Rates of specific peptide binding to the glycopeptide antibiotics vancomycin, ristocetin and avoparcin. J. Am. Chem. Soc. 110:1285-1286.

17. Sheldrick, G. M., P. G. Jones, O. Kennard, D. H. Williams, and G. A. Smith. 1978. Structure of vancomycin and its complex with acetyl-D-alanyl-D-alanine. Nature (London) 271:223-225.

18. Shlaes, D. M., A. Bouvet, J. H. Shlaes, C. Devine, S. Al-Obeid, and R. Williamson. 1989. Inducible, transferable resistance to vancomycin in Enterococcus faecalis A256. Antimicrob. Agents Chemother. 33:198-203.

19. Waltho, J. P., J. Cavanagh, and D. H. Williams. 1988. Aspects of molecular recognition: use of a truncated driven pseudoNOESY experiment to elucidate the environment of intermo- 
lecular electrostatic interactions in vancomycin. J. Chem. Soc. Chem. Commun. 1988:707-709.

20. Williams, D. H., and J. P. Waltho. 1988. Molecular basis of the activity of antibiotics of the vancomycin group. Biochem. Pharmacol. 37:133-141.

21. Williamson, R., S. Al-Obeid, J. H. Shlaes, F. W. Goldstein, and D. M. Shlaes. 1989. Inducible resistance to vancomycin in
Enterococcus faecium D366. J. Infect. Dis. 159:1095-1104.

22. Zeiger, A. R. 1988. Vancomycin prevents $\beta$-lactam antibiotics from causing soluble peptidoglycan secretion by Staphylococcus aureus cells, p. 563-567. In P. Actor, L. Daneo-Moore, M. Higgins, M. R. J. Salton, and G. D. Shockman (ed.), Antibiotic inhibition of bacterial cell surface assembly and function. American Society for Microbiology, Washington, D.C. 